\title{
Formando planificadores latinoamericanos. Estructuras institucionales en Uruguay y Chile en la década del sesenta
}

\section{Training Latin American planners. Institutional structures in Uruguay and Chile in the 1960s}

\author{
Alejandra Monti \\ ORCID: https://orcid.org/0000-0002-3182-6994 \\ Consejo Nacional de Investigaciones Científicas y Técnicas (CONICET), Rosario, Argentina. \\ Correo electrónico: montialejandra@gmail.com
}

Esta investigación fue financiada por Fondecyt № 3170311 y CONICET.

\section{Resumen}

El presente artículo indaga acerca de las formas que adquirió en América Latina - particularmente en Uruguay y Chile- la discusión en torno a la formación de profesionales desde un enfoque integral en el campo de la planificación urbana y regional, en el contexto de las políticas desarrollistas de mediados de la década del sesenta. Analizando comparativamente la actividad del Instituto de Teoría de la Arquitectura y Urbanismo en Uruguay y el Comité Interdisciplinario de Desarrollo Urbano en Chile, a través de fuentes primarias institucionales y fuentes secundarias referidas a la historia de la disciplina en la región, se avanza en la identificación de los vínculos entre Estado, universidad y técnica. Desde esta perspectiva, se reconoce que las dos experiencias actuaron como espacios de formación de cuerpos profesionales capaces de dar respuesta a las nuevas necesidades de los Estados, tanto en términos de ideas como de gestión del territorio, entendido este a partir de su rol como variable de las políticas desarrollistas para orientar el equilibrio nacional.

Palabras clave

Chile, formación, planificadores regionales y urbanos, Uruguay

\begin{abstract}
This article explores the debate raised for the training of professionals from an integral approach in urban and regional planning, and focused on Latin America - particularly in Uruguay and Chile - for the developmentalist policies of the mid-1960s. A comparative approach on the activity of the Instituto de Teoría de la Arquitectura y Urbanismo in Uruguay and the Comité Interdisciplinario de Desarrollo Urbano in Chile is proposed including primary and secondary data analysis for this discipline in the region and the identification of the links between State, university and technique. From this perspective, it is recognized that the two experiences acted as spaces for the formation of new professionals capable of responding to the needs of the States in terms of both ideas and territorial management, the latter understood from its role as a variable in developmental policies to guide national equilibrium.
\end{abstract}

\section{Keywords}

Chile, training, urban and regional planners, Uruguay 


\section{Introducción}

Repensar los vaivenes de la disciplina regional y urbana en América Latina, particularmente en el Cono Sur, enfrenta a la investigación a una lectura que, lejos de ser monolítica, asume variaciones interpretativas vinculadas como las propias trayectorias de los países o, como bien sostiene Alicia Novick, "se propone pensarlos como constelaciones en las que se entrecruzan ideas técnicas, modos de acción profesional y formas de regulación e intervención estatal que influyen sobre las agendas técnicas, las políticas y las sociales” (2004, p. 5).

Tomando como casos de estudio dos centros de formación profesional en planificación regional y urbana en el Cono Sur: el Instituto de Teoría de la Arquitectura y Urbanismo (ITU) en Uruguay y el Comité Interdisciplinario de Desarrollo Urbano (CIDU) en Chile, se sostiene que las estrategias pedagógicas aplicadas en la diagramación de sus programas de formación responden de manera directa a los nuevos requerimientos del Estado y, por consiguiente, a la necesidad de generar cuerpos técnicos formados bajo los preceptos de la planificación para el desarrollo.

Para ahondar en el contexto específico del estudio, se presenta aquí una descripción teórica que tiene a la formación de profesionales como eje de indagación. Esta lectura avanza en el reconocimiento de las geografías de incidencia en América Latina, visibilizando un primer momento ampliamente monopolizado por la academia norteamericana, seguido de la aparición de actores supranacionales $\mathrm{u}$ organismos profesionales que instalan el debate en el ámbito local. Allí se observa que lejos de ser entendido como un problema de traducción de ideas, el propio medio regional reconoce la necesidad de indagar sobre las lógicas a seguir en materia de formación de los profesionales.

A continuación, se reconstruyen las experiencias de los centros a partir de sus trayectorias institucionales, identificando la estructuración de sus programas de formación y los vínculos con el Estado. Para concluir se retoman, desde una perspectiva comparativa, los hitos centrales de las dos experiencias a fin de identificar las coincidencias y divergencias en las interpretaciones acerca de cómo se debe formar a un planificador regional y urbano en América Latina.

\section{Metodología}

Este trabajo se inserta en las historias de las disciplinas y las profesiones, en particular en la del planeamiento regional y urbano en América Latina. Arturo Almandoz sostiene que en los años de la segunda posguerra se produce un giro disciplinar que se traduce en una "manifestación del relevo y desplazamiento de los polos de Europa a Estados Unidos" (2019, p. 20) y, por consiguiente, la aparición de nuevos instrumentos de planificación que van a implicar además la renovación técnica (la formulación de proyectos de regionalización, el uso de la estadística y las encuestas), institucional (formación de centros de investigación con programas de posgrado, redes académicas, trabajos de asesorías a organismos estatales y supranacionales) y metodológica (con la interdisciplina como su rasgo más definitorio) de una disciplina que avanza a la región como ámbito de aplicación.

El escrito, expone los resultados de una revisión teóricohistórica compuesta principalmente por fuentes primarias recuperadas del Archivo Gómez Gavazzo y su página web en Uruguay ${ }^{1}$, el archivo de la Fundación Ford en los Estados Unidos y el archivo inédito del CIDU en Chile.

Entre los materiales utilizados se destacan las cartas personales, informes de actividades, informes financieros, publicaciones temáticas de los centros y documentos institucionales en un arco temporal que abarca desde 1955 hasta 1967.

El abordaje centrado en el documento ha permitido reconstruir las estrategias de acción de los centros en materia de formación de planificadores regionales y

1 Ver fadu.edu.uy/itu. 
urbanos, aportando a la discusión en torno a la historia disciplinar en el Cono Sur. Los centros analizados participaron del debate internacional sobre el rol de los planificadores desde un posicionamiento regional que, lejos del epicentro anglosajón de discusión (Inglaterra y Estados Unidos), planteó los límites y las expectativas de las renovadas relaciones entre planificación y burocracia estatal.

La elección de los dos centros analizados: el Instituto de Teoría de la Arquitectura y Urbanismo (ITU) en Uruguay y el Comité Interdisciplinario de Desarrollo Urbano (CIDU) en Chile, responde al reconocimiento de la actividad de estos en modelos estatales unitarios, identificando estrechos vínculos entre Estado y la universidad.

Tras una larga trayectoria, a partir de 1952, el ITU transformó su estructura y objetivos para estar en sintonía con las transformaciones de la disciplina, introduciendo teorías y modelos que fueron precisándose en el tiempo. El CIDU en cambio, es uno de los últimos centros en conformarse en la región, situación que lo ubica en un lugar privilegiado en tanto observador de las experiencias anteriores.

En esta línea, el análisis comparativo de estas dos experiencias permite identificar sus actuaciones como espacios de reflexión referidos a la formación de los planificadores, sopesando la articulación entre la actividad desde el ámbito académico y el desempeño en las nuevas oficinas de planificación en las esferas estatales. Una mirada acerca de los vínculos entre los espacios universitarios de cada país y las respectivas estructuras políticas permite reconocer los modelos particulares de articulación entre planificación y burocracias estatales. En ambos casos las universidades desempeñan un rol central, aunque con matices diferenciados, tanto en términos institucionales como en la propia comprensión del paradigma planificador desarrollista.

\section{Repensar la profesión. Discusionesentorno a los planificadores en América Latina}

El impacto de la segunda posguerra fue transcendental a escala global en tanto redistribuyó el poder político, económico y técnico. El desplazamiento de la centralidad de Europa hacia los Estados Unidos se tradujo en la disciplina urbana, en un cambio de referentes respecto del mundo anglosajón que involucró también una renovación metodológica e institucional, tanto en términos académicos como de la burocracia estatal. Sin embargo, este corrimiento no se produjo de forma abrupta, sino que paulatinamente ocurrió una traslación de la noción de urbanismo a la de idea planificación. Si el primero refiere a una matriz francesa anclada en lo urbano como escala de actuación y en el método científico como elemento de análisis; la planificación es entendida como una técnica racional de toma de decisiones tendiente a la transformación, donde la matriz económica se reconoce como una variable fundamental junto con los factores políticos, sociales y territoriales.

Autores como Peter Hall y Mark Tewdwr-Jones (2011), Nigel Taylor (2010) o Andreas Faludi (1978) han relevado las implicancias de las transformaciones de las ideas y teorías referidas a la planificación urbana, reconociendo en el período el surgimiento de una nueva disciplina a escala regional. Estos estudios, centrados en el mundo anglosajón, advierten los paralelismos entre las experiencias inglesas y norteamericanas, aunque reconocen sus variaciones en función de las estructuras gubernamentales y normativas en cada país. Pero lejos de ser entendido como un reemplazo de un concepto por otro, el desplazamiento de referentes, ideas y metodologías también se produjo en el medio latinoamericano, condición que es analizada desde la década del ochenta por la historiografía local (Almandoz, 2009, 2018; Novick, 2004; Pavez Reyes, 1992; Rigotti, 2014) desde una perspectiva que abandona una visión centrada en las relaciones entre "centro y periferia" por una comprensión y ampliación de las traducciones y reinterpretaciones que tuvieron asidero tanto en el mundo de las ideas como en su traducción práctica.

Arturo Almandoz (2018) le atribuye a una entusiasta visión de la sociología funcionalista el cambio disciplinar, identificando al trinomio industrialización, urbanización y desarrollo, como característico de las agendas políticas del período y condicionantes de un modelo propositivo que diagramó cambios epistemológicos, profesionales 
y académicos de fuerte impronta norteamericana. Esta evolución no se presenta análoga y sincrónica en el sur del continente, sino que responde a tradiciones propias y a retroalimentaciones entre actores locales y externos, condición que imposibilita una lectura monolítica del proceso en pos de las dislocaciones temporales y las matrices de referencia.

Sin embargo, se reconoce que, desde mediados de la década del cincuenta, se produjo un cambio de escala de actuación, donde la realidad urbana dio paso a la organización del territorio. La ciudad como objeto de estudio y como área de intervención de los urbanistas amplía sus horizontes a la región y a los territorios nacionales convirtiéndose en una variable de los programas desarrollistas.

Así, la emergencia de una nueva profesión asociada a la gerencia y proyecto del territorio se presenta vinculada con estructuras burocráticas más complejas y con organismos internacionales que van a actuar en el Cono Sur, a partir de programas de financiación y asesoría técnica y económica. Este escenario inaugura la necesidad de formar nuevos profesionales capaces, desde un enfoque integral, de "prestar una amplia gama de servicios a los organismos públicos en el campo del desarrollo urbano y regional" (Fundación Ford, 1968, p. 44). Pero cabe preguntarse, ¿qué significa formar profesionales desde un enfoque integral en el campo de la planificación urbana y regional? Y más aún, ¿qué implica hacerlo desde y para América Latina?

Estas reflexiones, lejos de iniciarse en el seno de las propias instituciones académicas o estatales nacionales fueron esbozadas en los años cincuenta en el expandido medio académico norteamericano como respuesta a la creciente relevancia que fue adquiriendo América Latina como espacio de acción profesional. El dinamismo norteamericano traducido en la apertura de centros universitarios que tienen como objeto de estudio el sur del continente, sumado a la presencia de estudiantes de posgrado latinoamericanos en sus programas académicos, transformaron las formas de diagramar la enseñanza de la planificación y sus objetivos con el fin de dar respuesta a un ampliado campo de acción referido no solo a los Estados, sino también a los nuevos organismos supranacionales que comenzaron sus trabajos de asesorías en el continente.

En 1957 se publica Education for Planning: City, State, and Regional (Perloff, 1970), texto fundante de la discusión sobre la formación de los planificadores y la definición de la planificación regional como campo de estudio. En esta obra, Perloff avanza en el reconocimiento de los países subdesarrollados como espacio propicio para la consolidación de la nueva disciplina regional, conjugando una renovación de los aparatos burocráticos con nuevos programas de financiación del desarrollo a escala nacional y regional, e identificando a los Estados como generadores de nuevas demandas de profesionales "presionando a las instituciones educativas a dar respuestas a estas condicionantes" (Friedmann, 1973, p. 299).

En la misma línea, John Friedmann sostiene que existe una expertise técnica que es la base de las habilidades analíticas de la educación en planificación. Sin embargo, esta consciencia técnica necesaria no debe soslayar "la relación entre técnica y poder político” (Friedmann, 1973, p. 305). En cuanto a la técnica, refiere a la organización de un sistema de recolección y análisis de la información, tanto económico como social, condición que precisa un abordaje interdisciplinario que permita aproximarse al análisis espacial de manera relacional (Friedmann, 1963), mientras que los vínculos con el poder político hacen referencia a la capacidad de los planificadores para influir en la toma de decisiones referidas al devenir del territorio y, por lo tanto, su cercanía a los espacios de poder. En el artículo "El planeamiento regional como disciplina de estudio", John Friedmann y Bernard Frieden (1963) le otorgan especial atención al rol de los planificadores y a la importancia de su formación académica, particularmente para los casos de estudiantes originarios de los países en desarrollo que realizan su formación profesional en los Estados Unidos y los estudiantes norteamericanos que brindarán servicios de asesoramiento en las regiones menos favorecidas del globo. Sostienen que la responsabilidad de los Estados Unidos en la formación de generaciones de planificadores supera las fronteras nacionales, siendo 
necesario adecuar los programas de estudio a fin de dar respuestas a las denominadas sociedades en transición. Más allá de posicionar el rol académico central de Norteamérica respecto de sus vecinos continentales (y de todos los países subdesarrollados en general), el artículo de 1963 no avanza en una identificación de las coordenadas para la formación de los profesionales planificadores, operando más como una hoja de ruta que como un programa de acción.

A estas indagaciones desde la academia norteamericana se le suman las propuestas de organismos supranacionales que refieren a la creación de centros dedicados al planeamiento en América Latina. En esta dirección la Unión Panamericana propuso a la OEA, a través del Programa de Cooperación Técnica, la creación de Institutos Regionales de Estudios Avanzados en Planeamiento Urbano en el marco de un proyecto denominado Proyecto $\mathrm{N}^{\circ} 23$. Sus funciones se orientan a la consolidación de un programa de estudio de dos años de duración para obtener un grado profesional en planeamiento regional y urbano, que sintetizará tareas de asesoramiento y asistencia técnica al Estado, cursos de formación, proyectos de investigación grupales e individuales, como así también la publicación y difusión de textos y trabajos de investigación.

En paralelo, en el medio latinoamericano, fueron surgiendo debates sobre la formación de los planificadores, tanto en los circuitos académicos como en organismos o sociedades profesionales. En 1958, la Sociedad Colombiana de Planificación (SCP), con el apoyo del Centro Interamericano de Vivienda y Planeamiento (CINVA), organizó la "Primera Reunión Nacional de Oficinas de Planes Reguladores”. La discusión acerca de la enseñanza de la planificación urbana se estableció a partir de la creación de un Instituto de Urbanismo que brindaba un curso de planificación para posgraduados articulando conocimientos históricos, físicos, técnicos, socioeconómicos, legales y administrativos. Sin hablar de interdisciplina, la Comisión de Estudios y Enseñanza de la SCP reconoció la colaboración de múltiples campos de conocimiento en el hacer de la planificación y el urbanismo, advirtiendo que "el país carece de suficiente personal preparado en estas dos ramas" (Sociedad Colombiana de Planificación, 1958, p. 43).

Es en la convivencia del uso de los términos urbanismo y planificación en los informes donde se visualiza que, todavía para finales de la década del cincuenta, no se presentaba tan clara esta distinción otorgándole al urbanismo la escala urbana y a la planificación la escala nacional/ regional. Se advierte además la continuidad de la enunciación del método racional de toma de decisiones como constitutivo de la elaboración de planes reguladores, una hibridación entre instrumentos disciplinares del urbanismo científico, pero con enfoques referidos a los nuevos temas/problemas del desarrollo y a las nuevas escalas de actuación (nacional y regional).

En 1961, la Sociedad Interamericana de Planificación (SIAP) dio cuenta del estado de la planificación en América Latina con la publicación de La enseñanza de la planificación en América Latina. El informe realizado por la misión técnica plantea la necesidad de generar programas de enseñanza de la planificación ajustados a la pregunta de "cómo mejorar la planificación del desarrollo económico y social de los países latinoamericanos" (SIAP, 1961, p. 01). Esta manifestación temprana de los objetivos del informe es indicativa de la prédica de los postulados de la planificación desarrollista, donde el territorio se convierte en una de las variables para el cambio, junto con la economía, la política y lo social, mientras que la planificación es entendida como un modo de actuar que "permite establecer relaciones de equilibrio entre los fines y medios" (SIAP, 1961, p. 6). La propuesta reconoce

tres niveles a los cuales el proceso del planeamiento debe ser aplicado: nacional, regional, y urbano; y debe incluir cursos orientados al planeamiento para el desarrollo nacional, regional y urbano, sumado a actividades de investigación, programas de extensión y educación pública y asistencia técnica (SIAP, 1961, p. 8).

Un punto central del documento detalla el rol otorgado a la universidad como institución líder del "movimiento de desarrollo económico-social” (SIAP, 1961, p. 22), 
siendo esta la encargada de preparar y capacitar a los profesionales, funcionarios y auxiliares técnicos necesarios para llevar adelante los nuevos programas del Estado. Si bien el informe resalta su rol, el mismo le asigna una vital importancia a la reorganización de los centros de planificación, independizándolos de las jerarquías de carreras existentes sugiriendo la innovación mediante la creación de centros líberos que eviten las deficiencias organizativas del sistema tradicional, facilitando así el trabajo multidisciplinario.

Cabe destacar que la mayoría de los países latinoamericanos para 1965 contaba con espacios académicos de formación en las técnicas de planificación, promoviendo una discusión al interior de las escuelas en torno al rol de los planificadores y sus vínculos con el Estado. Es allí donde se produce la mayor generación de centros a imagen y semejanza de los promulgados por la misión técnica de la SIAP, aportando un ciclo institucional en el que la planificación regional y urbana ya se presentaba como un campo disciplinar definido, aunque todavía volátil como puede verificarse en la década posterior.

Se han analizado hasta aquí las diferentes aristas que adquiere la discusión sobre la formación de profesionales planificadores en América Latina. El lugar que ocupa este debate en el ámbito académico norteamericano en los años posteriores a la consolidación de la Alianza para el Progreso (1961), permite identificar la asistencia técnica como una de las líneas de acción para su programa. Los planteos en torno a las necesidades de un nuevo tipo de profesional que sea capaz de incorporarse a las nuevas estructuras estatales se instalaron como una preocupación para los académicos y políticos locales, las entidades profesionales, las sociedades nacionales y regionales, marcando el pulso de las discusiones de la planificación en clave desarrollista en la región.

La imposibilidad de una lectura lineal de estas discusiones y sus polos de producción hizo visible la crisis de una interpretación sustentada en el par "importaciónexportación”, advirtiendo la necesidad de visibilizar estos procesos de transformación disciplinar desde una posición multivectorial y no unidireccional (Novick,
2004), donde el rol y destino de la formación de los profesionales planificadores en la región fuera discutido tanto en ámbitos externos como propios.

\section{Formando planificadores en el Cono Sur (1961-1967)}

Para dar cuenta de esta lectura comparada de los centros analizados: ITU y CIDU, se avanza en el análisis particular de las condiciones organizativas de cada uno de los ellos sumado al estudio de sus propuestas académicas y los vínculos entre programas teóricos y asistencia técnica, con el fin último de reconstruir ambas experiencias y aportar nuevas lecturas a la discusión sobre la formación de los planificadores en el período.

\section{Uruguay: Instituto de Teoría de la Arquitectura y Urbanismo}

El Instituto de Teoría de la Arquitectura y Urbanismo (ITU) funciona como centro asociado de la Facultad de Arquitectura de la Universidad Nacional de la República, Uruguay. Transformó su funcionamiento en 1952 producto de la reforma de los planes de estudio y, bajo la nueva dirección del arquitecto Carlos Gómez Gavazzo, introdujo el desplazamiento interpretativo de la disciplina que, aunque mantuvo el término urbanismo, dio comienzo a "la necesaria inclusión de otra ciencia que enfocara en las problemáticas rurales (el ruralismo), fundamentando una nueva disciplina: el Planeamiento Territorial" (De Souza, 2016, p. 388).

Es a partir de esta reformulación que se definen los alcances de sus actividades, asumiendo como objetivo "desarrollar tareas investigativas sobre el medio nacional a fin de fundamentar y reorientar la enseñanza de la profesión" (Instituto de Teoría de la Arquitectura y Urbanismo [ITU], 1973, p. 2). En esta línea, el planteo organizativo se sustenta sobre cuatro actividades principales: asistencia técnica, investigación, divulgación y, por último, administración. Estas se constituyen como las bases para crear un grado de consciencia técnica sobre los temas/problemas de la comunidad, "introduciendo la ampliación y especialización del campo profesional” (ITU, 1973, p. 3). 
Figura 1.

Esquema síntesis de la evolución de la disciplina urbana propuesto por Gómez Gavazzo.

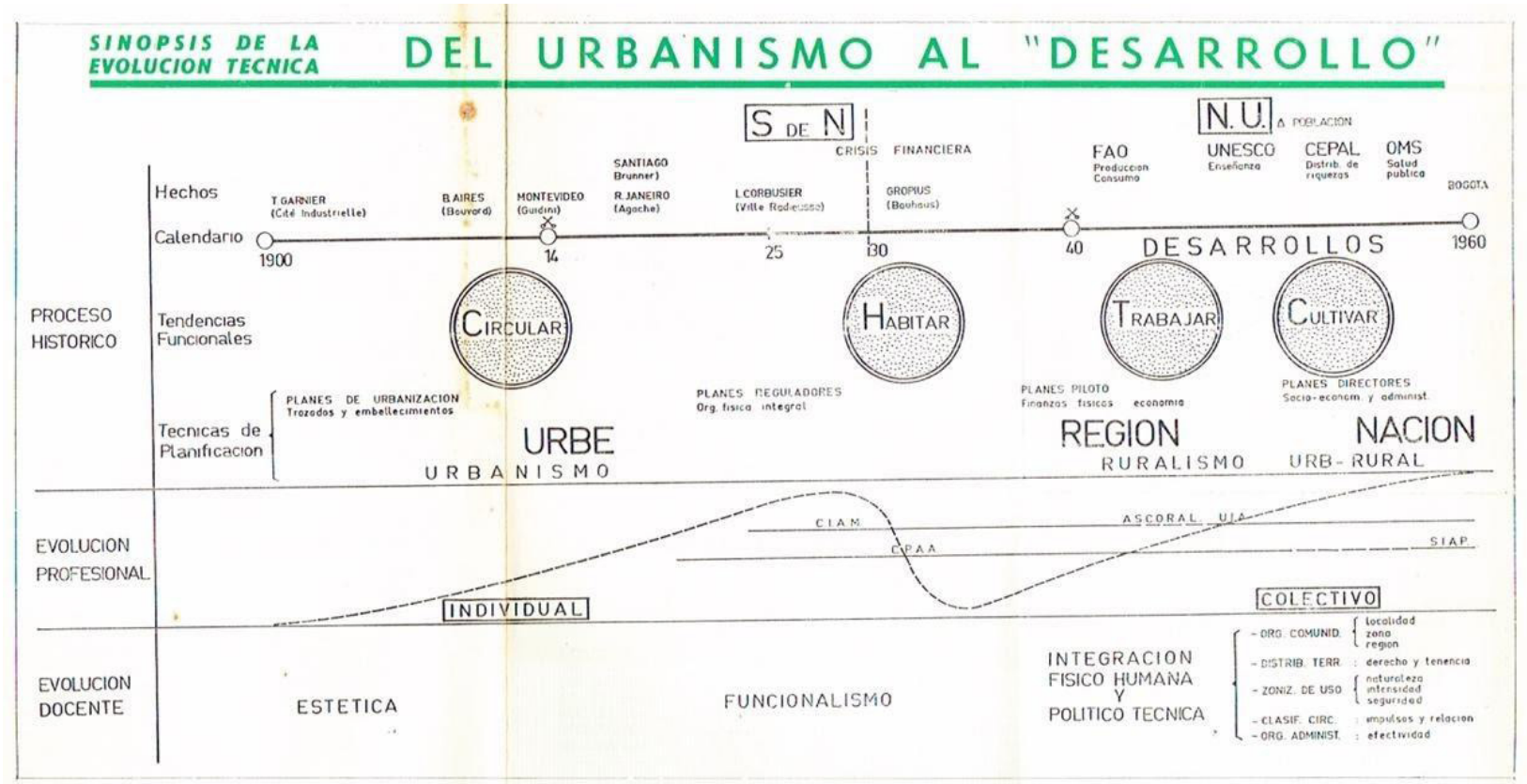

Nota. ITU, 1962b.

La afección de Gómez Gavazzo por teorizar la evolución de la disciplina urbana queda reflejada en el esquema denominado "Sinopsis de la evolución Técnica del urbanismo al desarrollo" (Figura 1) (ITU, 1962b, 08) en el cual, como bien señala De Souza (2016), no solo historiza los cambios conceptuales de la disciplina urbana, sino que también identifica en los diferentes períodos el rol de los profesionales y la evolución de la enseñanza de la disciplina.

Son los temas del momento caracterizados como desarrollo los que interesa remarcar en este escrito, examinando la consolidación de un nuevo modelo de evolución docente que abona una mirada colectiva desde la interrelación entre integración física-humana $\mathrm{y}$ político-técnica, haciendo referencia a un abordaje completo del territorio que, sin ser llamada planificación, asume sus instrumentos de acción.

En esta línea, el ITU asume un papel particular del enfoque profesional referido a la planificación urbana para el Uruguay, consolidando su rol como coordinador de las cátedras del área de arquitectura y urbanismo (ITU, 1959, s.p.), en el marco de la Facultad de Arquitectura. Esta tarea, denominada asistencia técnica interna, constituye uno de sus ejes principales de acción, advirtiendo su importancia dentro de la estructura de pregrado en términos de renovación de los temas y escalas de actuación, así como también los nuevos enfoques teóricos-metodológicos que asume la complejidad de la integración entre los aspectos físicos, sociales y políticos.

Para 1962, el esquema de objetivos y actividades del ITU se complejiza al generar un cruce entre investigación, enseñanza y cultura, que entiende a la docencia a partir de "asistir y orientar, en las materias de la especialidad, la labor técnica y educativa de las cátedras, para la formación de arquitectos" (ITU, 1962a, p. 1), aunque mantiene el esquema de asesoría y coordinación de años anteriores.

La particularidad del ITU se presenta en sus cursos para posgraduados, denominados también de Adiestramiento en Planeamiento Territorial. Destinados a graduados universitarios nacionales o extranjeros, se prevé en las convocatorias la posibilidad de incorporar en esta modalidad a funcionarios públicos que ejerzan sus tareas en oficinas municipales o nacionales. Lejos de configurarse como un curso teórico-metodológico de posgrado en línea con los esquemas de la OEA o la SIAP, el ITU adscribe a un programa que articula, por un lado, la asistencia a los cursos regulares de la Facultad de Arquitectura que el 
Figura 2.

Esquema institucional del ITU.

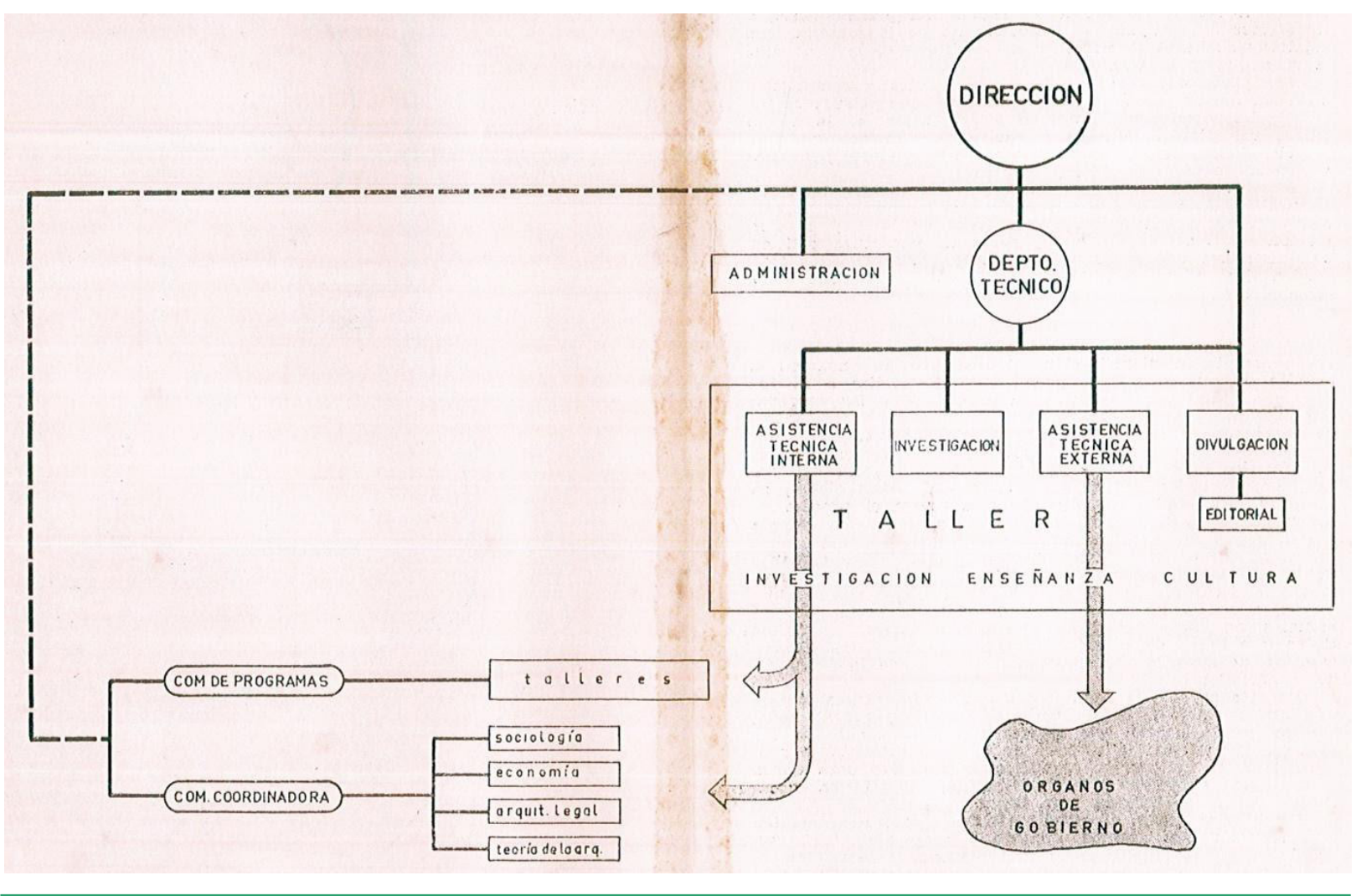

Nota. ITU, 1962a.

instituto coordina en calidad de observador (un curso) y activo (dos cursos); y, por el otro, la complementación de la formación con las tareas de investigación y asistencia técnica al Estado que el ITU lleva adelante (participación en un equipo de investigación, trabajos de asistencia técnica a municipios, investigaciones relativas a la asistencia a las cátedras y la actividad en investigaciones sobre temas de integración de planes con organismos nacionales y municipales), demostrando un posicionamiento que entiende a la formación de los profesionales desde una perspectiva práctica y asociada a las nuevas estructuras gubernamentales vinculadas con el desarrollo del territorio desde una mirada integral (Figura 2).

La asimilación de la formación de posgrado con trabajos de asistencia técnica refuerza los vínculos entre política y técnica, a partir de su comprensión como espacio de articulación entre formación, investigación y acción, que tiene como objetivo "coordinar y encauzar el proceso ejecutivo de la autoridad, orientar la opinión pública y extraer de la vida real el motivo cierto de la preparación profesional" (ITU, 1962a, p. 3).
Son innumerables los trabajos que realiza el ITU en los diferentes estratos del Estado, desde la preparación de expedientes comunales (Pantanoso, Sarandí del Yí, La PazLas Piedras); la asesoría a los gobiernos departamentales (Canelones, Colonia, Maldonado, Paysandú, Tacuarembó, Treinta y Tres, etc.); el asesoramiento al Instituto Nacional de Colonización y al Instituto Nacional de Vivienda Económica; el asesoramiento a los consejos de escala nacional (planificación de rutas y de centros de enseñanza primaria), a ministerios (planificación y recuperación de poblaciones indígenas y la planificación asistencial de la salud pública), así como también actividades de asesorías permanentes o de carácter integral a gobiernos departamentales que requieren los servicios del ITU (Río Negro, Artigas, Cerro Largo, etc.).

Como es posible observar, mientras el programa de asistencia técnica interna (enseñanza) se mantiene constante desde 1952 hasta 1965, no variando las asignaturas coordinadas (Sociología, Economía, Arquitectura Legal y Proyecto Arquitectónico) ni el alcance de la asesoría, la multiplicidad de los trabajos de asistencia técnica 
externa muestra la amplitud de los temas trabajados por el ITU, sumado a otorgarle a la universidad un rol distintivo dentro de las estructuras de la función pública.

Es en parte por la propia dimensión del Uruguay y la impronta de su universidad pública más importante, que resulta posible fundamentar el rol constitutivo del ITU como espacio de discusión teórico-práctico de la planificación en el país. Si la formación de los profesionales se constituye como objetivo de acción, esta no se presenta aislada del campo de trabajo. A pesar de que la educación de los arquitectos incorpora ciertas asignaturas vinculadas con escalas de abordaje superadoras del objeto arquitectónico y la ciudad, la misma se desarrolla a la manera tradicional; mientras que para convertirse en planificadores, los alumnos de posgrado deberán tomar los cursos específicos digitados por el ITU e incorporarse a los trabajos de asistencia técnica y de investigación asociados, con el fin de adquirir la expertise necesaria para dar respuestas a las nuevas necesidades del territorio y a los requerimientos del Estado en sus múltiples capilaridades.

\section{Chile. Centro Interdisciplinario de Desarrollo Urbano (CIDU)}

En marzo de 1966 se iniciaron las actividades del Comité Interdisciplinario de Desarrollo Urbano (CIDU) con sede en la Pontificia Universidad Católica de Chile (PUC). Su creación se presenta en un momento particular del devenir político-institucional del país, que bajo la presidencia del demócrata cristiano Eduardo Frei Montalva, inició un proceso de "renovación de las agendas políticas, respondiendo conscientemente a los modelos de desarrollo" (Purcell, 2014, p. 71). La puesta en marcha del CIDU y su funcionamiento entre 1966 y 1970 estuvo definida por los aportes de la Fundación Ford (FF) dentro de un programa de más largo alcance que esta entidad filantrópica desarrolló en el país, denominado Programa de Asesoría en Desarrollo Urbano y Regional de Chile (Urdapic, 1965-1970). Entre los objetivos fundamentales enunciados por el programa se encontraba "el de crear las capacidades institucionales necesarias para actuar eficazmente frente a los cambios generados por el proceso de urbanización que está experimentando el país" (Fundación Ford, 1968, p. 2 ), condición que plantea la acción de asesoría dentro de la burocracia estatal a escala nacional (Oficina de Planificación Nacional, Odeplan) y local (Ministerio de Vivienda y Urbanismo, Minvu), sumado a la capacitación técnica que tiene al CIDU y a la Universidad Católica como espacio de acción.

Fernando Quesada sostiene que la instalación de la sede institucional de la FF en Santiago (1963) consolidó su acción en el país, marcada por "acontecimientos políticos, procesos sociales y culturales, a los que la FF no fue refractaria” (2015, p. 112). En la misma línea, Inderjeet Parmar afirma que la FF quería trasplantar en Chile un modelo científico-social a la manera norteamericana, orientado a la conformación de cuerpos técnicos que pudiesen proporcionar "asesoramiento técnico basado en la experiencia profesional, independientemente de la ideología política” (2015, p. 191). Si bien la acción de la FF en Chile constituye un tema de más largo recorrido interesa remarcar, a los fines de este artículo, las condiciones centrales para la comprensión de la creación del CIDU en el marco institucional de la PUC y dentro de un programa más complejo de asesoría estatal. Pese a que la Universidad de Chile (UCH) se constituyó como la mayor institución universitaria chilena receptora de fondos y subsidios para proyectos y programas de la FF durante la década del sesenta, ya desde 1962 la PUC contaba con grants en diferentes campos del saber (The Ford Foundation Office for Argentina and Chile, 1963) que incluían también el asesoramiento a la transformación institucional de la Universidad Católica a partir de un programa de desarrollo (Watson, 1963, p. 1).

En este marco de asesoría universitaria y asistencia técnica al Estado demócrata cristiano, la creación del CIDU se constituyó como una experiencia piloto en materia de planeamiento regional y urbano de cuño desarrollista, sintetizando en su estructura institucional y sus postulados teóricos-conceptuales las premisas enunciadas por la misión técnica de la SIAP en 1961. Como respuesta al 


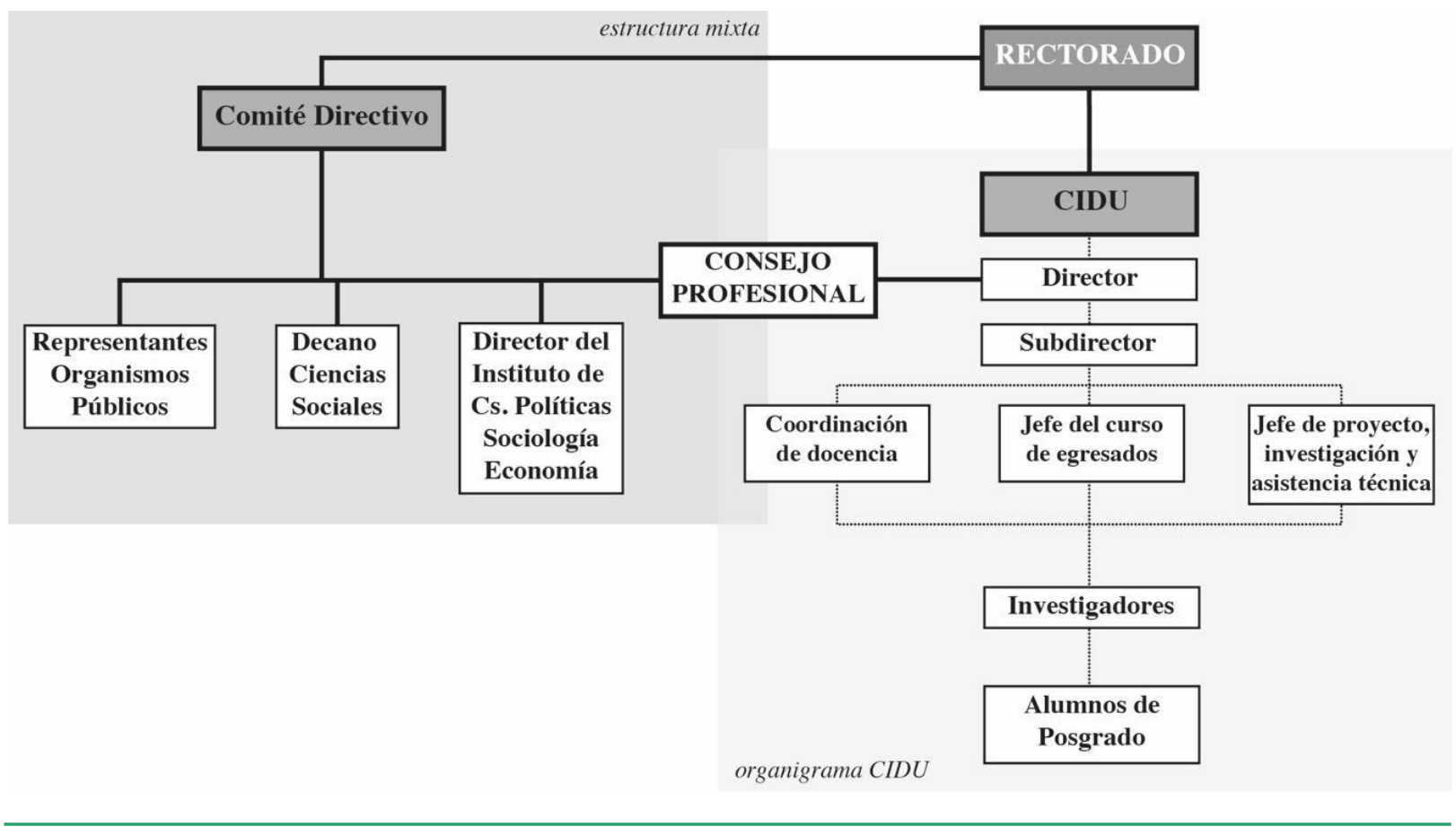

Nota. Elaboración propia a partir del material de archivo.

proceso de cambio, la planificación es entendida como la "técnica profusamente utilizada en toda oportunidad en que se pretende obtener la máxima eficiencia en la utilización de los recursos con el fin de alcanzar ciertas metas en un período determinado" (CIDU, 1966a, p. 1).

El objetivo del CIDU es formar especialistas que enfrenten los complejos problemas del desarrollo desde la "concurrencia de técnicas de análisis y de instrumentos de acción en donde intervengan diferentes disciplinas" (CIDU, 1966a, p. 01). Su funcionamiento como comité durante el primer año, independiente del accionar de la Escuela de Arquitectura y vinculado directamente con la Rectoría, permite confirmar desde un posicionamiento institucional, el rol asignado a la universidad como espacio de capacitación orientado a la acción con sentido social hacia la consecución del bien común. Es desde esta figura de centro flotante que el CIDU plantea su estructura de manera independiente, en pos de la generación de un tipo de centro que articula diferentes disciplinas. Esto se lleva adelante con la conformación de un nuevo sistema de enseñanza que permite la complementación de los conocimientos básicos a partir del diálogo con otras profesiones, en definitiva, una visión interdisciplinaria del problema del desarrollo (Figura 3).
Su programa de acción se encamina a

promover y realizar programas de investigación interdisciplinaria orientados a la solución integral de los problemas del desarrollo; sumado a ampliar la dotación del país de especialistas en planificación en relación con los diferentes aspectos económicos, sociales y físicos, de este desarrollo y de proporcionar asesoría técnica interdisciplinaria de alto nivel al Gobierno e instituciones locales y regionales (Fundación Ford y Universidad Católica de Chile, 1965, p. 03).

Para ello identifica tres áreas de actuación para el nuevo centro: enseñanza, investigación y asistencia técnica.

En referencia a la enseñanza, las actividades durante el primer año se canalizaron en dos líneas de trabajo: una serie de cursos que se desarrollan para los alumnos del pregrado, asociados a las escuelas de leyes, arquitectura e ingeniería y a los centros de investigaciones sociológicas e investigaciones económicas que funcionaban en la PUC; en segundo lugar, se presentan los cursos básicos del CIDU, dictados en sus instalaciones por los investigadores del centro y destinados, durante 1966, a los alumnos 
Figura 4.

Cursos dictados por el CIDU entre 1966/1967.

\begin{tabular}{|c|c|c|c|c|c|}
\hline & \multirow{2}{*}{ CURSOS } & \multicolumn{2}{|c|}{1966} & \multicolumn{2}{|c|}{1967} \\
\hline & & $1^{\circ}$ semestre & $2^{\circ}$ semestre & $1^{\circ}$ semestre & $1^{\circ}$ semestre \\
\hline \multirow[t]{4}{*}{$C I D U$} & \multirow[t]{2}{*}{ Básicos } & \multirow{2}{*}{$\begin{array}{c}\text { Urbanización y } \\
\text { desarrollo } \\
\text { económico y } \\
\text { social } \\
\text { Friedmann - Geisse } \\
\text { CIDU } \\
\end{array}$} & \multirow{2}{*}{$\begin{array}{c}\text { Teoria y } \\
\text { Método de } \\
\text { Planificación } \\
\text { Friedmann - Geisse } \\
\text { CIDU } \\
\end{array}$} & $\begin{array}{c}\text { Urbanización y métodos } \\
\text { de planificación } \\
\text { económico y social } \\
\text { CIDU } \\
\end{array}$ & $\begin{array}{l}\text { Teoria y métodos de } \\
\text { la planificación } \\
\qquad \mathrm{CIDU} \\
\end{array}$ \\
\hline & & & & $\begin{array}{l}\begin{array}{l}\text { Desarrollo } \\
\text { económico }\end{array} \\
\\
\quad \mathrm{CIDU} \\
\end{array}$ & $\begin{array}{l}\begin{array}{l}\text { Sociología } \\
\text { urbana }\end{array} \\
\\
\text { CIDU } \\
\end{array}$ \\
\hline & Introductorios & $\begin{array}{l}\text { Evolución de } \\
\text { las ciudades }\end{array}$ & $\begin{array}{l}\text { Organización } \\
\text { del desarrollo }\end{array}$ & $\begin{array}{l}\text { Introducción a } \\
\text { la economía } \\
\text { ECONOMIA } \\
\end{array}$ & $\begin{array}{l}\text { Organización del } \\
\text { desarrollo } \\
\text { LEYES }\end{array}$ \\
\hline & & ARQ. & LEYES & $\begin{array}{l}\text { Evolución de las } \\
\text { ciudades } \\
\\
\end{array}$ & $\begin{array}{c}\text { Aspectos políticos } \\
\text { del desarrolloo } \\
\text { LEYES }\end{array}$ \\
\hline \multirow[t]{3}{*}{$\begin{array}{l}\text { Relacionados } \\
\text { con Escuelas } \\
\text { o Facultades }\end{array}$} & \multirow{3}{*}{ Opcionales } & \multirow[t]{3}{*}{$\begin{array}{l}\text { Programación de } \\
\text { vivienda y equip. } \\
\text { comunitario }\end{array}$} & \multirow[t]{3}{*}{ Estadística } & $\begin{array}{c}\text { Análisis de } \\
\text { Sistemas } \\
\text { INGENIERIA } \\
\end{array}$ & $\begin{array}{r}\text { Estadística } \\
\text { ECONOMIA } \\
\end{array}$ \\
\hline & & & & $\begin{array}{c}\text { Problemas de } \\
\text { población }\end{array}$ & $\begin{array}{l}\text { Transporte } \\
\text { urbano }\end{array}$ \\
\hline & & & & SOCIOLOGIA & INGENIERIA \\
\hline
\end{tabular}

Nota. Elaboración propia a partir del material de archivo.

que cursaban el último año de la carrera. Esta primera prueba piloto de funcionamiento proponía para 1967 la formalización de los cursos de posgrado y el inicio de las actividades del programa interdisciplinario, contando con un alumnado que ya había ampliado su experiencia académica en los nuevos abordajes de la planificación, pudiendo realizar su tesis bajo el auspicio del CIDU, transformándose en un curso de posgrado (Figura 4).

En los programas de las asignaturas dictadas por el CIDU se establece la matriz teórica conceptual propuesta por el centro, entendiendo al desarrollo urbano a partir de la relación entre procesos de urbanización e industrialización y de urbanización y evolución económica-cultural del país. Estas formulaciones no solo refuerzan la necesidad de un abordaje interdisciplinario, sino que en su fundamentación subyace una interpretación del territorio y el proceso de urbanización como variable de procesos interrelacionados más complejos vinculados con las decisiones políticas, los factores económicos y las transformaciones sociales. El dictado de los cursos del CIDU contaba con los aportes del grupo de expertos norteamericanos que integraban el Udapic (John Friedmann y Lawrence Mann), sumado a los profesores del CIDU (Guillermo Geisse y Ricardo Jordán).
Las tareas de docencia son complementadas, bajo la figura de la asistencia técnica, con una serie de cursos de extensión o de entrenamiento de personal intermedio en ejercicio, que reformulan los vínculos entre Estado, universidad y planificación regional y urbana. El objetivo es "satisfacer las necesidades inmediatas de capacitación de personal intermedio, realizando labores de desarrollo urbano" (CIDU, 1966b, p. 2). El carácter de estos es intensivo y orientado a la enseñanza de técnicas y métodos de uso práctico que entreguen herramientas para la materialización de programas de desarrollo urbano.

El primer curso de extensión se realizó en 1966 a pedido del Minvu y en colaboración con la Facultad de Economía de la Universidad de Chile y se denominó Curso de Programación del Desarrollo Comunal y contó con la participación de 35 técnicos municipales que realizaban labores en planificación urbana en Chile (Friedmann, 1966, p. 3). El objetivo era preparar a los estudiantes (funcionarios públicos) para el trabajo en oficinas de programación municipal que el Minvu ya había establecido (10), o bien intentaba crear en el próximo año.

En 1967, el CIDU impartió un curso corto referido a la programación de las inversiones regionales para 
planificadores, financiado por la recientemente aprobada Odeplan, el cual contó con la asistencia de 25 funcionarios estatales. En 1968, se dictó el curso de Planificación Regional para funcionarios de Odeplan y oficinas de planificación del sector público (25 alumnos), sumado a un curso de Desarrollo Comunal en Áreas Metropolitanas destinado a funcionarios de las municipalidades de las tres áreas metropolitanas del país: Santiago, Valparaíso y Concepción (32 alumnos), además de un curso sobre conceptos y técnicas de la planificación social, destinado a las instituciones privadas chilenas que trabajan en la promoción del desarrollo social.

En estos primeros años de funcionamiento, se visualiza un modelo de aproximación a la enseñanza de la planificación que articula, por un lado, la noción de la planificación como un método racional de toma de decisiones; $y$, por el otro, la articulación entre formación académica y formación profesional asociada a las necesidades del Estado. Esta última condición, no puede separarse de la renovada estructura institucional de la burocracia estatal chilena durante el gobierno de Frei Montalva, generando nuevos cruces que redefinen el rol del CIDU no solo como formador de nuevos profesionales versados en las renovadas técnicas de planificación, sino también la capacitación de los técnicos a escala municipal y nacional, diferenciando las temáticas en función de las escalas de actuación.

\section{Análogos, pero no homólogos}

La revisión de las estrategias de los dos centros frente a la formación de planificadores durante la década del sesenta presenta una lectura institucional que permite trazar ciertas particularidades y también analogías entre ambas experiencias.

En primer lugar, se identifican diferencias en las temporalidades de las trayectorias institucionales de los centros. El ITU es uno de los primeros centros universitarios referidos a la disciplina urbana de América Latina (1937), mientras que el CIDU (1966) se consolida a mediados de la década del sesenta, y se convierte en uno de los últimos exponentes de centros diagramados bajo la impronta desarrollista. A pesar de esto se enfrentan a la misma pregunta: ¿cómo formar profesionales en las nuevas técnicas y prácticas de la planificación?

Las respuestas a este interrogante se plantean desde la propia estructura institucional y su vinculación entre el pregrado y el posgrado. Mientras que la estrategia del ITU es intervenir y coordinar un grupo de asignaturas del pregrado, identificando que para el posgrado las actividades que completan la formación están orientadas a la praxis más que a la teoría. En esta línea, la formalización del programa de posgrado no se presenta de la manera tradicional y siguiendo los parámetros internacionales, sino que plantea que la asistencia a los cursos de pregrado y las tareas de asistencia técnica e investigación que llevan adelante los integrantes del instituto les otorgan a los profesionales las habilidades necesarias para desempeñarse en las diferentes escalas de la planificación.

El CIDU asume durante el primer año de funcionamiento un modelo que articula pregrado y posgrado de manera estable, sumado a una serie de cursos de formación técnica destinados y requeridos por los organismos estatales. En este sentido, el CIDU plantea cierta analogía con el ITU, identificando en la articulación entre niveles educativos una operatoria que le permite la colaboración de las estructuras de las facultades, escuelas e institutos de la Universidad Católica. Sin embargo, este enlazamiento se diferencia a partir de la identificación de su organigrama dentro de la estructura de la PUC, que reconoce su dependencia directa del Rectorado y, por lo tanto, la autonomía en la toma de decisiones tanto programáticas como financieras. Durante el segundo año de funcionamiento, las diferencias con el centro uruguayo se acrecientan, ya que la condición de centro flotante e independiente del CIDU, independiza a su vez los temas/problemas de los cursos dictados por el centro que tienen a la urbanización, el desarrollo y la planificación como eje de indagación, mientras que ciertos abordajes referidos al plano legal, arquitectónico, sociológico e ingenieril siguen siendo trabajados por cada una de las facultades. Pero, si todavía en 1968 la apuesta institucional se articulaba a partir de los vínculos entre pregrado y posgrado, con la formalización del Curso 
de Egresados sobre Planificación del Desarrollo Urbano Regional, el CIDU orienta su actividad a los "estudios del proceso de urbanización y de las técnicas para el diseño de políticas públicas tendientes a su orientación" (CIDU, 1968, p. 05). El curso está destinado a egresados de varias disciplinas, e incorpora tareas de taller (investigación) además del desarrollo de una tesis orientada al campo de investigación del alumno, condición que termina por establecer el programa de posgrado.

Desde esta perspectiva, se reconocen criterios diferenciados en la formación de los planificadores en cada instituto, ya que para el ITU la práctica y la investigación constituyen la mayor carga horaria para los participantes del posgrado, mientras que para el CIDU el programa completo de formación requiere un mínimo de 40 créditos $^{2}$ distribuidos en tres trimestres, más la realización de la tesis. Si la asistencia técnica externa es la base programática de la formación de nuevos planificadores en el ITU, el CIDU apuesta por los cursos teóricos como armazón estructural del programa, contando con la activa participación de planificadores como John Friedmann, Lawrence Mann, Ralph Gakenheimer o René Eyheralde, etc; que no formaban parte del CIDU, sino que del staff de la FF, operando en el CIDU como asesores invitados. Los profesionales del ITU, en cambio, eran profesores e investigadores pertenecientes al plantel de la facultad, que trabajaban como asesores del Estado uruguayo en las tareas de asistencia técnica desde el ITU.

A partir de esta diferencia, es posible destacar un punto crucial para la comprensión del rol de los centros y sus planificadores en cada país. En dos estructuras de gobierno centralizadas, que iniciaron en los años sesenta un proceso de modernización de la burocracia estatal referida a la organización del territorio en clave de variable de transformación, se advierte una coincidencia en la retroalimentación entre política, técnica y universidad. Sin embargo, si la Universidad Católica a través del CIDU
Figura 5.

Estructura territorial de la función pública en Uruguay.

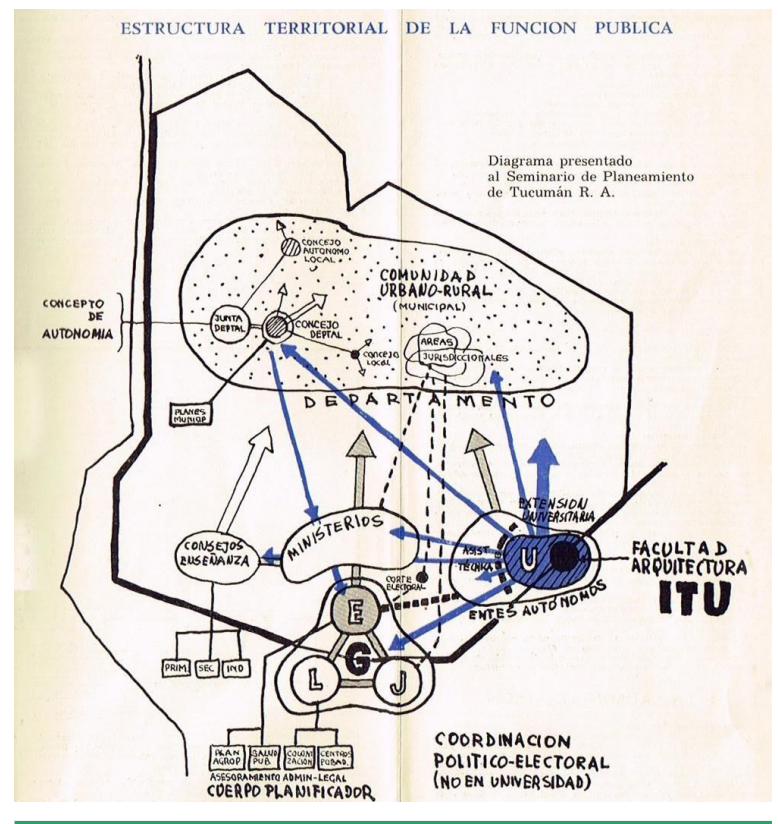

Nota. ITU, 1962a.

es entendida como un "dador de insumos generales al sistema", es decir, provee el marco institucional para la formación de los planificadores alineada con los requerimientos del Estado, el ITU se presenta como el espacio de acción a través de una cantidad considerable de trabajos de asistencia técnica externa. Esta idea queda plasmada en los esquemas de organización propuestos por sus referentes institucionales. Gómez Gavazzo le otorga gran centralidad a la Facultad de Arquitectura y al ITU, destacando la asistencia técnica y la extensión universitaria como ejes del accionar de la facultad y, a partir de allí, establece los vínculos con el poder político y técnico desde una perspectiva centrada en la autonomía del accionar del ITU (Figura 5).

La propuesta de organigrama, realizada por John Friedmann desde el Urdapic, plantea una condición distinta de los vínculos entre universidad y Estado, situando al CIDU en un esquema más amplio de colaboración que articula diferentes niveles de la burocracia coincidentes con escalas de actuación territorial: Odeplan para la escala nacional, la Oficina de Planificación Regional (Orplan) para las regiones y el Minvu en la escala urbana

2 El CIDU organiza su funcionamiento a través de créditos que constituyen unidades de medida del trabajo académico. La propuesta de 40 créditos refería al programa en su totalidad que se realizaría en un promedio de 13 cursos (cuatro cursos introductorios, seis básicos y tres de especialización), además del taller y tesis. 
Figura 6.

Organigrama de colaboración de la Fundación Ford en Chile 1965-1970.

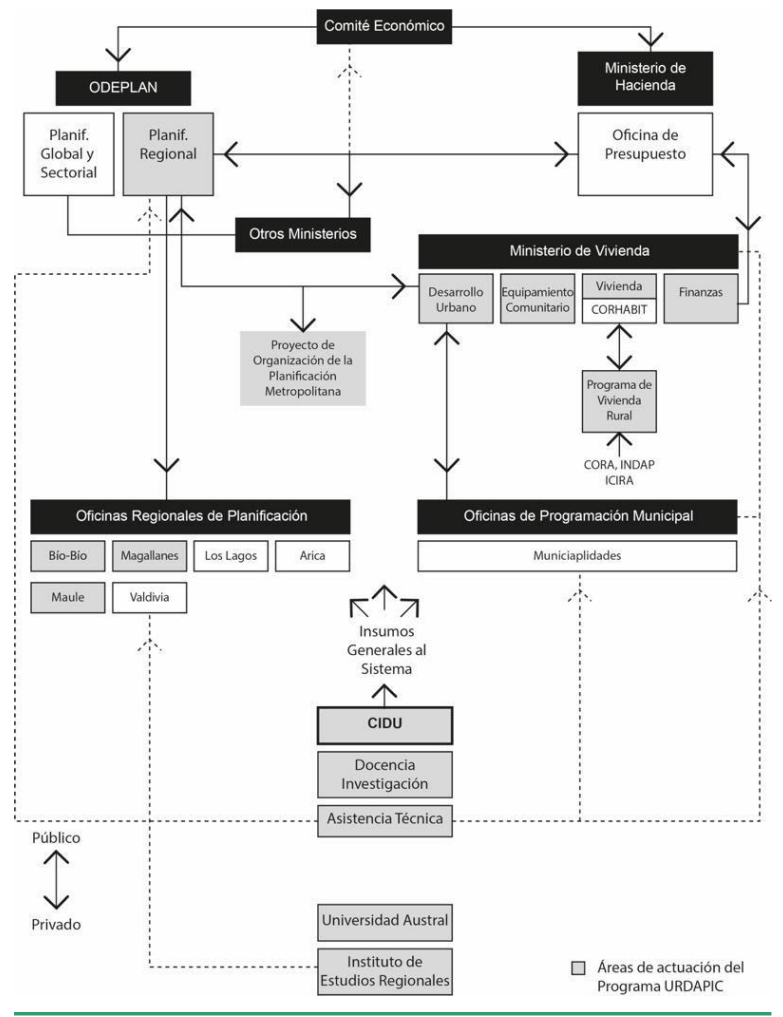

Nota. Fundación Ford, 1968.

y arquitectónica. Esta estructura ubica al CIDU como un espacio de formación exento de la estructura del Estado, pero en amplia colaboración con este a partir de la asistencia y alineación con los objetivos temáticos y la agenda vinculada al desarrollo (Figura 6).

\section{Conclusiones}

Evitando realizar un juicio de valor sobre cuál modelo institucional y programa de formación respondió de mejor manera a las necesidades de la época y del país, interesa reconocer que detrás de cada propuesta pedagógica de los centros, existió una estrecha vinculación entre los programas de formación y la generación de oficinas técnicas estatales que requerían profesionales formados en las nuevas técnicas de la planificación.

Esta coincidencia convierte al ITU y al CIDU en exponentes de la transformación de la disciplina en el Cono Sur, pues generaron programas de formación de posgrado que tienen a la planificación regional y urbana como horizonte. Esta idea pone en relieve el rol del territorio como una de las variables para lograr el equilibrio territorial característico de los anhelos desarrollistas, incorporando en el debate las variables políticas, sociales y principalmente económicas. Así, tanto el ITU como el CIDU, plantean la necesidad de una mirada interdisciplinaria sobre la renovada disciplina urbana, que evidencia la incorporación de diferentes escalas de actuación. Los trabajos de asesoría externa en el ITU y los cursos externos destinados a agentes del gobierno chileno en el CIDU dan cuenta de una mirada multiescalar asociada a las oficinas estatales de planificación.

Cabe destacar también, el rol central que adquiere la universidad como espacio de producción científica y técnica. En Uruguay, esta centralidad es aún más evidente, siendo la Universidad Nacional de la República la mayor y más respetada casa de altos estudios del país y, por lo tanto, el espacio primordial de formación de profesionales. En Chile, la tradición universitaria ofrece mayores variables, reconociendo a la Universidad de Chile, la Universidad Católica de Chile y la Universidad de Concepción como las tres principales estructuras universitarias para 1960. En este sentido, los vínculos entre el programa político de Eduardo Frei Montalba y la Democracia Cristiana con la Fundación Ford, sumado a la acción de actores relevantes para la gestión del centro (Sergio Larraín y René Eyheralde) permiten la radicación del CIDU en la PUC, por sobre la Universidad de Chile.

Frente a estas observaciones, y reconociendo que los ideales desarrollistas tuvieron matices interpretativos en cada país de la región, se sostiene que, en la trayectoria de más larga duración del ITU y sus protagonistas, subyace un proceso de cambio disciplinar que va incorporando paulatinamente las nuevas ideas y modelos de trabajo y financiación asociados a organismos supranacionales y asociaciones profesionales regionales, situación que le otorga al espacio universitario una centralidad en el desarrollo de las tareas de asesoría. El CIDU, en tanto, surge en el apogeo de la expansión de la asistencia técnica e institucional de la Fundación Ford en Chile, y en un momento de consolidación de la planificación regional y urbana a escala latinoamericana. Esta aparición tardía en el escenario latinoamericano cuenta con la ventaja del conocimiento de las experiencias previas de los centros de formación, investigación y asistencia técnica de la región, 
sumado a un aporte sustancial para su funcionamiento y consolidación por parte de la FF por un período de cinco años, situación que le otorga grandes ventajas institucionales a la hora de diagramar su proyecto académico de formación profesional de planificadores regionales y urbanos.

Desde esta perspectiva, se reconoce que, frente a los desafíos institucionales de cada país, sus estructuras de gobierno y sus esquemas burocráticos de organización, los centros y sus actores operaron como vectores de la transformación disciplinar adecuando sus estrategias pedagógicas y sus esquemas organizativos a las nuevas necesidades de los Estados, tanto en términos de ideas como de gestión del territorio. ${ }^{44}$

\section{Referencias bibliográficas}

Almandoz, A. (2009). Mudanças politicas e institucionais para o planejamentolatino-american o dosegundo pós-guerra. http:// books.scielo.org/id/wvqr3/pdf/gomes-9788523209261-09.pdf

Almandoz, A. (2018). Modernization, Urbanization and Development in Latin America, 1900s-2000s. Routledge.

Almandoz Marte, A. y Monti, A. I. (2019). De urbanistas a planificadores: A\&P Continuidad, 6(11), 18-25. https:// doi.org/10.35305/23626097v6i11.225

Comité Interdisciplinario de Desarrollo Urbano, CIDU (1966a). El programa de docencia e investigación interdisciplinaria de la Universidad Católica de Chile. Base para publicación en "El Mercurio". Autoedición.

Comité Interdisciplinario de Desarrollo Urbano, CIDU (1966b). Pre informe sobre programa de cursos. 1966. Autoedición.

Centro Interdisciplinario de Desarrollo Urbano, CIDU (1968). CIDU. Programa 1968 (p. 14) [Programa de actividades]. Universidad Católica de Chile, Autoedición.

De Souza, L. (2016). Imaginarios rurales: el modelo de afincamiento en la planificación rural del Uruguay de Gómez Gavazzo. Labor Engenho, 10(4), 383-401. http:// dx.doi.org/10.20396/labore.v10i4.8646249
Faludi, A. (1978). Essays on Planning Theory and Education. Pergamon.

Friedmann, J. (1963). Introducción al estudio y práctica de la planificación (Ficha IPRUL Na8). Archivo IPRUL.

Friedmann, J. (1966, octubre 14). Evaluación de actividades del CIDU [Carta a John Nagel]. CIDU.

Friedmann, J., \& Frieden, B. (1963). Regional Planning as a Field of Study. Journal of the American Institute of Planners, 29(3), 168-175. https://doi.org/10.1080/01944366308978061

Friedmann, J. R. P. (1973). Urbanization, Planning, and National Development. Sage Publications.

Fundación Ford (1968). Informe anual 1967-68. Autoedición.

Fundación Ford y Universidad Católica de Chile (1965). Bases para un doble acuerdo a celebrarse entre la Universidad Católica de Chile y la Fundación Ford. Archivo CIDU.

Hall, P. G., \& Tewdwr-Jones, M. (2011). Urban and Regional Planning. Routledge.

Instituto de Teoría de la Arquitectura y Urbanismo, ITU (1959). Boletín Informativo $N^{\circ} 16$. Resumen de las actividades del ITU en el año 1958. Universidad de la República, Facultad de Arquitectura; Archivo ITU - Uruguay.

Instituto de Teoría de la Arquitectura y Urbanismo, ITU (1962a). Boletín Informativo $N^{\circ} 22$. Resumen de las actividades desarrolladas en el año 1961. Universidad de la República, Facultad de Arquitectura; Archivo ITU - Uruguay.

Instituto de Teoría de la Arquitectura y Urbanismo, ITU (1962b). Boletín Informativo $N^{\circ} 23$. Planificación y Reforma Agraria en el Uruguay. Universidad de la República, Facultad de Arquitectura; Archivo ITU - Uruguay.

Instituto de Teoría de la Arquitectura y Urbanismo, ITU (1973). Informe sobre directivas para el cumplimiento de funciones asesorales por parte del I.Y.U (Expediente N³1544; p. 10). Facultad de Arquitectura; Archivo Gómez Gavazzo. 
Novick, A. (2004, mayo 28). Historias del urbanismo/ historias de la ciudad. Una revisión de la bibliografía. Seminario de Crítica. Instituto de Arte Americano e Investigaciones Estéticas, 137. http://www.iaa.fadu. uba.ar/publicaciones/critica/0137.pdf

Parmar, I. (2015). Foundations of the American Century: The Ford, Carnegie, and Rockefeller Foundations in the Rise of American Power. Columbia University Press.

Pavez Reyes, M. I. (1992). La institución del urbanismo en la Facultad de Arquitectura y Urbanismo de la Universidad de Chile (1928-1988) (1º). Departamento de Urbanismo de la FAU - U. Chile. http://repositorio.uchile.cl/ handle/2250/118077

Perloff, H. S. (1970). Education for Regional Planning in Less Developed Countries. Settlement Study Centre.

Purcell, F. (2014). Guerra Fría, motivaciones y espacios de interacción. El caso del Cuerpo de Paz de Estados Unidos en Chile, 1961-1970. En T. Harmer y A. Riquelme (Eds.), Chile y la Guerra Fría global (pp. 71-88). RIL editores.

Quesada, F. (2015). La universidad desconocida: el convenio Universidad de Chile, Universidad de California y la Fundación Ford (1a ed.). Editorial de la Facultad de Filosofía y Letras de la Universidad Nacional de Cuyo.
Rigotti, A. M. (2014). Las invenciones del urbanismo en Argentina 1900-1960: inestabilidad de sus representaciones científicas y dificultades para su profesionalización (A\&P). UNR Editora, Editorial de la Universidad Nacional de Rosario. Facultad de Arquitectura, Planeamiento y Diseño.

Sociedad Interamericana de Planificación, SIAP. (1961). La enseñanza de la planificación en América Latina. Autoedición.

Sociedad Colombiana de Planificación (1958). Informe final: Primera Reunión Nacional de Oficinas de Planes Reguladores. Centro Interamericano de Vivienda (CINVA).

Taylor, N. (2010). Urban Planning Theory Since 1945. Sage.

The Ford Foundation Office for Argentina and Chile (1963). The Ford Foundation's program in Argentina and Chile (Box 448 FA739A; p. 24). Ford Foundation; Ford Foundation Records.

Watson, R. (1963, agosto 8). Santiago Inter Program Development (Box 7 Folder 309) [Carta a Joseph Rupert]. Rockefeller Foundation Records. 
